\title{
Auch das Beständigste war nicht mehr von Dauer!' Die Darstellung des Eigenen und des Gegenübers in Andreas Birkners Roma Die Tatarenpredigt
}

\author{
Maria SASS \\ Prof.Dr. Lucian-Blaga-Universität Sibiu/Hermannstadt. \\ E-Mail: maria.sass-zosim@web.de
}

\begin{abstract}
The present study focuses on imagology. Starting from the theoretical aspects of the concepts self-image and hetero-image, the analysis ponders upon the imagological constructs of two ethnical groups in the novel of the Romanian German-language author Andreas Birkner. In this analysis, the self image identifies with the one of the Transylvanian, and the image of the other is that of the Roma. The analysis of Birkner's novel leads to the conclusion that there have been certain mental images deeply rooted in historical reality and which can be, partly, explained by means of collective memory parameters. Stereotypes and prejudices should be considered in this context.
\end{abstract}

Key words: Andreas Birkner, imagology, self-image, hetero-image, stereotype, prejudice

\section{Einführung:}

Die Entwicklung der rumäniendeutschen ${ }^{2}$ Literatur außerhalb desbinnendeutschen Sprachraums, unterspezifischenhistorisch-

1 Birkner, Andreas: Die Tatarenpredigt. Roman. Europaverlag. Wien 1973, S. 190. Die weiteren Seitenangaben aus Birkners Roman werden in runden Klammern zu den Zitaten angeführt.

2 Der Begriff "rumäniendeutsche Literatur" bezeichnet das deutsche literarische Schrifttum auf dem Gebiete Rumäniens und umfasst die 
politischen, sozialen und kulturellen Bedingungen, hat ihr ein eigenes Profil verliehen. Die Vetreter dieser Literatur schöpfen ihre Stoffe und Themen aus einem regionalen Repertoire, doch in ihrer Darstellungsweise ist die Tendenz zur überregionalen Geltung zu gelangen und im deutschsprachigen Raum Anerkennung zu finden, nicht zu übersehen.

Zum Thema Imagologie bietet Siebenbürgen reichliches Untersuchungsmaterial, die im Zentrum Rumäniens gelegene Region weist eine multiethnische Struktur auf und hat - vom historischen, etnographischen und künstlerischen Standpunkt - eine besonders interessante Konfiguration. Die Beziehungen zwischen den jeweiligen Ethnien der siebenbürgischen Landschaft haben zur Konturierung von Eigen- und Fremdbilder geführt und in die Literatur Eingang gefunden.

\section{Zum Begriff "Imagologie"}

Die wissenschaftliche Beschäftigung mit einem Forschungsobjekt setzt klare Definitionen fachspezifischer Begriffe voraus. Für den Bereich der Imagologie ist diese Prämisse schwer zu erfüllen, weil in der Fachliteratur eine Unschärfe der Definionen sowie das Fehlen einheitlicher Begrifflichkeit herrscht, in vielen imagologischen Arbeiten ist ein synonymer oder austauschbarer Gebrauch von Begriffen festzustellen. Um Ungenauigkeiten zu vermeiden, erweist es sich von Bedeutung, die Termini, mit denen in der vorliegenden Arbeit gearbeitet wird, zu klären.

Die theoretische Grundlage meines Aufsatzes ist von der Studie Die Imagologie als Arbeitsbereich der Komparatistik ${ }^{3}$ von Funda Kiziler Emer gegeben, in der alle in der Textanalyse angewandten Begriffe definiert sind.

schriftstellerischen Produktionen aller Provinzen (Siebenbürgen, Banat, Bukowina), in denen Deutsche gelebt haben.

3 http://www.ijoess.com/Makaleler/1275068110_Die\%20Imagologie\% 20als.pdf: 6.05 .2019 
Das Wort Imagologie ist ein Kompositum, bestehend aus dem Wort "Imago" und dem Suffix "-logie". Das lat. Wort "Imago" bedeutet nichts anderes als "Bild" und "-logie" heißt "Kunde, Lehre, Wissenschaft von... ."4 Aus der etymologischen Definition geht hervor, dass die Imagologie ein Wissenschaftszweig (Arbeitsbereich der Kompartistik ${ }^{5}$ ) ist, der Bilder untersucht. Das Wort "Imago" findet man auch in der Psychoanalyse und bedeutet "im Unterbewusstsein Vorhandenes [Ideal]bild einer anderen Person der sozialen Umwelt. ${ }^{6}$ Daraus ist zu schließen, dass die Imagologie allgemein "Bilder vom anderen Land" behandelt, der Forschungsgegenstand ist vom "Fremden" gegeben. Logvinov machte darauf aufmerksam, dass das Bild "in der interkulturellen Kommunikation in Form von Stereotypen, Klischees oder Vorurteilen"' auftaucht. Die Vorstellungen von den Anderen sind als mentale Bilder aufzufassen, deren Entstehung oft historisch bedingt ist. Sie werden von Gustav Siebenmann folgendermaßen definiert "Die Bilder in unseren Köpfen, bezeichnet man als Imagotype." Bezieht sich das in unserem Kopf entstandene Bild auf die

4 Wahrig: Deutsches Wörterbuch. Bertelsmann Lexikon Verlag, Güterloh und München, 1986-1991, S. 682; 843. Zit. nach: Funda Kiziler Emer: Vgl. Fußnote 3.

5 Wilpert, Gero von: Sachwörterbuch der Literatur. Kröner Verlag, Stuttgart 1989, S. 405-406. Zit. nach: Funda Kiziler Emer: Vgl. Fußnote 3.

6 Duden:Deutsches Universalwörterbuch, A-Z. Dudenverlag, Mannheim/ Wien/Zürich, 1989, S.754. Zit. nach: Funda Kiziler Emer: Vgl. Fußnote 3.

7 Logvinov, Michail, I.: Studia Imagologica: zwei metodologische Ansätze zur komparatistischen Imagologie 1. In: Germanistisches Jahrbuch GUS: Das Wort, 2003, S. 203. Zit. nach: Funda Kiziler Emer: Vgl. Fußnote 3.

8 Siebenmann, Gustav: Methodisches zur Bildforschung. In: Gustav Siebenmann u. Hans-Joachim König (Hrsg.): Das Bild lateiamerikas im deutschen Sprachraum. Max Niemeyer Verlag, Tübingen 1992, S. 1. Zit. nach: Funda Kiziler Emer: Vgl. Fußnote 3. 
Selbstdarstellung, als Individuum oder als Gruppe, so ist dieses als Auto-Imagotype zu bezeichnen. Andererseits, im Falle der Bilder, die wir uns von den Anderen machen, sprechen wir von dem Hetero-Imagotype. ${ }^{9}$ Es ist hervorzuheben, dass das Bild des Anderen/ Hetero-Imagotype immer vom Bild des Eigenen /Auto-Imagotype abhängt, weil bei der Beurteilung der Alterität immer das Eigene zum Kriterium wird. ${ }^{10}$

In der vorliegenden Arbeit sollen, ausgehend von der angeführten theoretischen Grundlage zum Auto-Imange und HeteroImage, die von Andreas Birkner im Roman Die Tatarenpredigt konstruierten Bilder zweier ethnischen Gruppen untersucht werden, dabei wird als Eigenbild das der Siebenbürger Sachsen und als das Andere bzw. Gegenüber, das Romabild, betrachtet. Bei der Untersuchung der genannten literarischen Bilder werden sowohl werkimmanente, als auch außertextuelle Zusammenhänge ermittelt.

\section{Zum Autor}

Andreas Birkner, geboren am 15. August 1911, in einem am Fuße der Fogarascher Gebirge gelegenes siebenbürgisches Dorf, Kleinschenk, besuchte die Grundschule im Heimatdorf, danach das theologisch-pädagogische Seminar in Hermannstadt, das er 1930 absolvierte. Zeit seines Lebens wirkte er als Schriftsteller und Pfarrer. Birkner debütierte 1934 als Erzähler in der Kronstädter Zeitschrift Klingsor (1924-1939), in der er mehrere Prosaschriften veröffentlichte. 1941 publizierte er sein erstes Buch Die Straße neben dem Strom; 1944 erschienen der Roman Wind in der Tenne und die Erzählung Der gelbe Windhund. Seine letzte Veröffentlichung in Rumänien war die Novelle Aurikeln

9 Ebenda. Zit. nach: Funda Kiziler Emer: Vgl. Fußnote 3.

${ }^{10}$ Fischer, S. Michael: Nationale Images als Gegenstand vergleichender Literaturgeschichte. UNtersuchungen zu Entstehung der komparatistischen Imagologie. Bouvier Verlag, Bonn 1981, S. 46. Zit. nach: Funda Kiziler Emer: Vgl. Fußnote 3. 
(1956), für die ihm ein Preis der Zeitung Neuer Weg verliehen wurde. Er ist einer der fünf deutschsprachigen Autoren, die 1959 im Kronstädter Schriftstellerprozeß verurteilt wurden, Birkners Strafe betrug 25 Jahre Zwangsarbeit. 1964 wurde er aus der Haft entlassen und 1968 mit den übrigen Schriftstellern von den rumänischen Behörden rehabilitiert. ${ }^{11} 1966$ übersiedelte er nach Deutschland, wo er bis zu seiner Pensionierung als Krankenseelsorger in der Augenklinik von Freiburg arbeitete. Seit damals besuchte er nie mehr Rumänien. In Deutschland publizierte er hauptsächlich im Europaverlag (Wien, München, Zürich). Der Roman Die Tatarenpredigt erschien 1973 im erwähnten Europaverlag. Im selben Verlag wurden auch die anderen Romane Birkners Das Meerauge (1976), Heinrich, der Wagen bricht (1978), Spiele mit Nausikaa (1981) und die Erzählungen Der lange Segen und andere Geschichten (1975), Der Teufel in der Kirche (1980) veröffentlicht. Sein Nachlass wurde dem Institut für deutsche Kultur und Geschichte Südosteuropas anvertraut, wo er sich auch jetzt befindet. Einige seiner Erzählungen wurden unter der Bearbeitung von Hans Bergel $(* 1925)$ und Stefan Sienerth $(* 1948)$ im IKGS-Verlag München herausgegeben. ${ }^{12}$

Andreas Birkner hat ein umfangreiches Werk von unterschiedlichem literar-ästhetischen Wert geschaffen, das durch detailreiche Darstellungen dem Leser Einblicke in die Probleme seiner einstigen Heimat gewährt.

Über seine Art zu schreiben drückt er sich folgendermaßen aus: „Ich baue meine Geschichten nicht nach einem System, sie sind intuitiv und plötzlich da: ich brauche sie nur noch aufzuschreiben [...]. Konstruierte Geschichten taugen nach meinem

${ }^{11}$ Nowotnik, Michaela: „95 Jahre Haft”. Kronstädter Schriftstellerprozess 1959: Darstellungsformen und Deutungsmuster der Aufarbeitung. In: Halbjahresschrift für südosteuropäische Geschichte, Literatur und Politik. 24. Jg., Heft 1-2/2012, S. 173-181.

${ }^{12}$ Birkner, Andreas: Der Brautschmuck des Sebastian Hann. Erzählungen. Herausgegeben und mit einem Nachwort von Hans Bergel. Mit einem editorischen Bericht von Stefan Sienerth. München 2002. 
Dafürhalten nichts."13 Daraus kann geschlossen werden, dass er sich aus der Wirklichkeit inspiriert hat. Durch seine Schriften möchte er seinen Nachfolgern die siebenbürgische Vergangenheit in literarischer Form bekannt machen.

\section{Inhalt und Problematik des Romans}

Birkners Roman Die Tatarenpredigt bietet ein umfassendes Panorama Siebenbürgens vor dem Hintergrund der Nachkriegszeit und der sozialen Umwälzungen, die der Übergang zur sozialistischen Gesellschaft mit sich brachte, ausgehend von der Darstellung des Dorfes Pretai, in dem Sachsen, Rumänen, Ungarn, Juden, Armenier und Zigeuner, ja auch ein Krimtatar zusammen lebten. Jede ethnische Gruppe hatte ihre eigene Sprache, Religion, Kultur, ihre eigenen Sitten und Bräuche. Im Mittelpunkt der Darstellung befindet sich die ,nationale, konfesionelle und kulturelle Vielfalt des siebenbürgisch-sächsischen Dorfes. "14 Wie oben angedeutet spielt sich die Handlung des Romans in dem sächsischen DorfPretai (Bratei/bei Mediasch) $\mathrm{ab}$, doch als Schauplätze der Handlung werden auch andere siebenbürgische Orte (Bußd, Fogarasch, Mediasch, Hermannstadt) erwähnt.

Der Protagonist, Jonas Malmkroger, ein sächsischer Bauer, versucht, seinen verstorbenen Knecht, Göngür Mustafa, einen im Dorf verbliebenen Krimtataren, begraben zu lassen. Doch in der unmittelbaren Nachkriegszeit zeigt sich dieses Unternehmen als sehr schwierig, denn kein Priester betrachtet sich als zuständig, weil der "Tatter" Moslem ist: weder gehört er zur evange-

${ }^{13}$ Nowotnik, Michaela: „95 Jahre Haft”. Kronstädter Schriftstellerprozess 1959: Darstellungsformen und Deutungsmuster der Aufarbeitung. In: Halbjahresschrift für südosteuropäische Geschichte, Literatur und Politik. 24. Jg., Heft 1-2/2012, S. 173-181.

${ }^{14}$ Sienerth, Stefan: Andreas Birkner: ,"Vale Saxonia Septemcastrensis. " In: „Daß ich in diesem Raum hineingeboren wurde...” Gespräche mit deutschen Schriftstellern aus Südosteuropa. München 1997, S. 137. 
lischen, noch zur orthodoxen oder unierten Konfession. Malmkroger ging von einem Pfarrhaus zum anderen, um einen Pfarrer jedwelcher Konfession zu überzeugen, den "Tatter" zu begraben, und konnte nicht begreifen, wieso keiner seinen Knecht beerdigen wollte, "In Siebenbürgen. Nicht irgendwo in der Welt. In Siebenbürgen, dem ersten Land der Welt, das die Religionsfreiheit proklamiert hatte." (Birkner: 307)

Selbst Pfarrer, stattet Andreas Birkner alle Geistlichen sowohl mit menschlichen Schwächen, als auch mit Vorzügen aus. Doch wird hervorgehoben, dass in der gegebenen historischen Situation - unmittelbare Nachkriegszei und der Übergang zur sozialistischen Gesellschaft - alle Angst haben: "Sie haben Angst" (Birkner: 191), sagt der evangelische Pfarrer über die rumänischen Priester, und als Kommentar des Erzählers wird hizugefügt: "der Furchtsame versteht als erster den Ängstlichen... " (Birkner: 193)

Wie auch in anderen seiner Erzählungen oder Romane, wählt Birkner auch in der Tatarenpredigt eine realistische Schreibweise und verwendet als Darstellungsform die 'auktoriale Erzählsituation', dabei dominiert die 'Außenperspektive', der Narrator steht außerhalb des Universums seiner Figuren und gewinnt dabei 'epische Distanz'. Diese fast 'göttlich' anmutende Perspektive ermöglicht ihm die Schauplätze, gelegentlich die Zeiten seiner Geschichte durch Rückblenden und Vorausdeutungen zu wechseln. Der Erzähler verfügt souverän über Zeit und Raum und kann auch die Worte, Gedanken und Gefühle seiner Figuren nach Belieben ausbreiten, zusammenfassen oder verschweigen, dabei werden Wertungen oder Urteile formuliert und gelegentlich wird von der Möglichkeit Gebrauch gemacht, die Figuren oder deren Ansichten ironisch $\mathrm{zu}$ kommentieren. ${ }^{15}$ Das Ergebnis solcher Narration ist ein „Wirklichkeitseffekt" bzw. eine „Mimesis-Illusion,” aber auch

${ }^{15} \mathrm{https}$ ://www.uni-due.de/einladung/Vorlesungen/epik/auktorial.htm: 23.02.2014 
eine gelungene Erzählweise, die dem Leser große Freude bereitet kann, denn "Andreas Birkner verfügt in reichem Maß über die heute so selten gewordene Begabung des großen Erzählens. Sein Roman ist Beispiel für die Erneuerung einer bedeutenden Romantratidion."16

Die Analyse von Birkners Roman lässt auf die Existenz bestimmter mentaler Bilder schließen, deren Wurzeln zum Teil in der konkreten historischen Wirklichkeit verankert sind, die teilweise durch Parameter "des sozialen Gedächtnisses"17 erklärt werden können. Dabei spielen tiefverwurzelte Vorurteile und ein hohes Maß an Mythisierung eine nicht zu vernachläßigende Rolle. Der Autor nimmt sich nicht explizit vor, "das Andere" zu thematisieren, das Hauptthema ist die Darstellung der multiethnischenGesellschaftSiebenbürgens, dochkommtden "Fremdenbildern" eine wichtige Rolle zu. Als siebenbürgisch-deutscher Autor stellt er "Das Fremde innerhalb der eigenen Ordnung"18 dar, die "fremde" Welt der Zigeuner hat er "miterlebt" und konstruiert sie in der Narration nach.

\section{Das Eigenbild in Birkner Tatarenpredigt}

In Andreas Birkners untersuchten Roman ist das Autoimage, die sächsische Identitä $t^{19}$, die aus der Perspektive des Selbsterlebten dargestellt wird. Obwohl eine konkrete kurze Zeitepoche nachgestaltet wird, in Rückschau werden die Charakteristiken

${ }^{16}$ Klappentext: Birkner, Andreas: Die Tatarenpredigt. Roman. Europaverlag. Wien 1973

${ }^{17}$ Erzse-Boitor, Kinga: Das Bild des Anderen in der rumäniendeutschen Kinder- und Jugendliteratur. Peter Lang, Frankfurt am Main, 2009, S. 71.

${ }^{18}$ Ebenda, S. 125).

${ }^{19}$ Sächsische Identität: die Identität einer deutschsprachigen Gemeinschaft Siebenbürgens, Angehörige des Königsbodens, den sie autonom verwalteten und eine eigene Geschichte vom 13.-19. Jahrhundert hatten. 
dieser ethnischen Gruppe aufgenommen, wobei ein Bewußtseins- und Identitätswandel der Siebenbürger Sachsen deutlich wird. Für die Sachsen steht symbolisch der Protagonist Jonas Malmkroger, dessen Name nichts

...über Irdisch-Zerbrechliches [meint], (...) der Name Malmkroger bedeutete nur soviel, daß des Mannes Vorfahren aus dem siebenbürgischen Dorf Malmkrog stammten, wie die Bogatscher aus Bogatsch, die Foragascher aus Foragasch und die Meschendörfer aus Meschendorf. Und eigenmächtig, hölzern und unbiegsam war er nicht allein, in allen Dörfern lebten seine Geschwisterkinder. (Birkner: 54)

Er stellte das Herkömmliche dar: "Malmkroger war also einer, bei dem die Sitte noch im Schwange war"(Birkner:197), wenn er die Glocken der Evangelischen Kirche läuten hörte, betete er noch Herr, erbarm dich unser!, eine Sitte, die längst abgekommen war. Doch "Malmkroger war zuzeiten auch Spott zuzutrauen. Im vorliegenden Fall also, daß er den Herrgott anrief zum Beistand gegen die Pfarrer (Birkner: 197) In der Absicht, seinen moslemischen Knecht ordentlich zu begraben, durchzieht Malmkroger würdig die Dorfgassen bis zu Pfarrer Umling: "...immer schritt er würdig daher, was freilich nicht heißen soll, daß ihn die Würde als Last erdrückte." (Birkner: 74) Selbst von den anderen ethnischen Gruppen wird er positiv eingeschätzt. Der Bulibascha sieht in Malmkroger einen Traditionalist, der sächsische Bräuche einhält: "Ich hab dich tanzen sehen, sagte der Zigeuner. [...] Du tanzt anders als alle übrigen. [...] Altmodisch, ja, nickte Malmkroger, sie lachen alle über mich. Ich hab es so gelernt als ich jung war." (Birkner: 9) Im Volksrat fiel die Frage auf, ob sie den "Tatter" "wie einen Hund verscharren" (Birkner: 72) werden, die Folge ist, eine Charakterisierung der Sachsen:

Die Sachsen begraben ihre Toten nicht wie Hunde, wurde die Frau des Lehrers Olaru mit einmal Ernst, das kann man ihnen nicht nachreden. (...) Ja die eigenen Toten wohl nicht, trupfte der Mann auf, aber hier handelt es sich um einen Krimtataren. (Birkner: 72) 
In Retrospektive wird der zweite Weltkrieg erwähnt, in dem Malmkroger: "Beide Kinder verloren [hat] (...), den einen gegen die Russen, den anderen gegen die Deutschen." (Birkner: 52) Ins Gespräch wird auch die Enteignung gebracht, als die Sachsen ihre großen Häuser verloren hatten und bloß ein "Hinterstübchen" (Birkner: 20) bewohnen durften:

Alle übrigen Sachsen hatten über Nacht Haus, Hof, Grund und Boden und dazu alle Habe verloren und hausten seither, kaum geduldet, in Hinterstübchen, Sommerküchen, Backhaus und Ställen auf ihren eigenen Höfen, froh noch, wenn sie der neue Besitzer als Knecht oder Tagelöhner beschäftigte. (Birkner: 36)

Das war eine "himmelschreiende Ungerechtigkeit" (Birkner: 22), die "Anderen", hauptsächlich Zigeuner, besetzten die sächsischen Häuser und bekamen durch die Agrarreform, die von den Sachsen abgezwungenen Ackerflächen. "Ihn [Jonas M.], der in der Armee gedient, die gegen den Faschismus ausgezogen war, hatte das Gesetz von der Enteignung ausgenommen" (Birkner: 28)", doch es gab auch für ihn keine Ruhe mehr: "Von zwölf Milchkühen war eine einzige übriggeblieben. Die Steuern. Der Nachtrag zu der Nachtragsbesteuerung. Die Abgaben. Die Fleischquote. Die Heuquote. Die Getreidequaote. Die Milchquote. Die Kartoffelquote. Davon waren die Ställe leer geworden." (Birkner: 42) Auf seinem Weg von einem Pfarrer zum anderen und von Pretai nach Mediasch und zurück, reflektiert Malmkroger über die stark herabgekommene Gesellschaft, vor der er den Respekt verloren hatte. Selbst die Naturbeschreibung stellt Endzeitgefühle dar:

Das Laub des Baumes lag als ein runder Teppich auf der Erde (...) Langsam ging er unter den Linden des Kirchenwegs nach Hause. Knöcheltief watete er durch das herabgefallene Laub. Auch hier lag es auf der Erde. Keiner rechte es zusammen, um es als Streu zu verwenden. Die Sachsen hatten kein Vieh mehr in den Ställen. Die neuen Hofbesitzer dachten gar nicht daran. Vater Zeno hatte gemeint, die haben nur noch den Karl Marx und die Politik in ihrem Kopf. 
Vielleicht würde es eine Sächsin sammeln, doch es eilte nicht damit, es würde ihr kein Rumäne oder Zigeuner zuvorkommen. (Birkner: 198)

Nicht einmal Totenscheine werden ordentlich ausgestellt, eine Hebamme übernahm diese Tätigkeit und schrieb auf das Zeugnis als Grund des Todes Altersschwäche: "Altersschwäche, entschied der Kreisarzt, Fünfundachzigjährige sterben an Altersschwäche. " (Birkner: 70)

Leitmotivisch wird wiederholt, dass die Abgaben, die den Bauer ins Verderben führen, abgeliefert worden sind:

Die Quoten waren abgeliefert, die Heuquoten, die Weizenquote, die Gerstenquote, die Haferquote, die Milchquote, die Fleischquote, die Steuerquote bezahlt - sie hatten ihn auch dieses Jahr noch nicht untergekriegt. Würden sie mir einem Nachtrag zur Jahressteuer versuchen? Und danach mit einem Nachtrag zur Nachtragssteuer? Zwei Mastschweine hielt er für diesen Fall bereit. Danach war's dann aus, dann musste er das Rennen aufgeben. Danach mußte er ihnen Grund und Boden und alles Ackergerät überlassen. Auch das Pferdegespann. Der Volksrat brauchte für Dienstfahrten nach Mediasch und Bußd Wagen und Pferde. Der Amtsdiener würden sie kutschieren" (Birkner: 56)

Die Überlegenheit der Sachsen wird immer wieder betont, sogar wenn Jonas Malmkroger über das Zeitvergehen nachdachte. Es war Reif gefallen, "ein richtiges Weinlesewetter" (Birkner: 74). Der Bauer dachte über Zeiten nach, als die Weinlese Wochen lang gedauert hatte, doch "seit die Weinberge enteignet worden waren, waren die neuen Besitzer schon am ersten Tag fertig. Über die Hälfte der Weinberge waren eingegangen. In zehn Jahren war der Schaden nicht wiedergutzumachen. " (Birkner: 75).

Das Eigenbild wird auch durch einige negative Eigenschaften der Sachsen ergänzt. Die Burghütterin Zirr Kappes wird als Tratschfrau charakterisiert, sie "war eine Elster" (Birkner: 77), die sich in Angelegenheiten aller Leute einmischte und gut 
tratschen konnnte. Sehr kritisch wird der Pfarrer Umling dargestellt, weil er nicht bereit ist, den "Tatter" christlich zu begraben - "der Tatter, Malmkroger, ließ sich der Pfarrer endlich hören, der Tatter war nicht evangelisch." (Birkner:80), "ich kann nicht einen Moslem beerdigen!'”(Birkner: 81) - deshalb spricht Malkroger über die Angst und die "hasenherzige Feigheit des Pfarrers" (Birkner: 78). Das detaillierte siebenbürgische Bild der unmittelbarea Nachkriegszeit, das Birkner in der Tatarenpredigt konstruiert, wiedergibt eine stark verfallene Gesellschaft. Lüge und Verleumdung herrschte überall, sogar in den Institutionen; falsche Zeugen sind bereit mit der Hand auf der Bibel zu schwören, dass sie das eine oder andere gesehen haben. Uneheliche Kinder versuchen beliebige Väter unter den Prietsern zu suchen. Die ganze sächsische Dorfgemeinschaft lebte in einer großen Angst:

Nicht sogleich im August 1944, aber bald danach hatten die Sachsen ihre Tore verschlossen, nach den Deportationstagen im Januar 1945 verschlossen die Zurückgebliebenen die Tore. Als ob ihnen das etwas geholfen hätte. Es half ihnen gar nichts. Hinter verschlossenen Toren lebt keiner sicherer. Er lauert Tag und Nacht auf Geräusche und Bewegungen vorm Tor. Ein Bekannter klopft oder kratzt leise ein verabredetes Zeichen. Sogar dem mißtrauten sie. Nicht einmal die Kinder dürfen laut reden bei verschlossenen Toren - schweigt doch, ich höre ja nichts, ob jemand am Tor ist! Und sie schweigen erst recht, wenn's laut ans Tor klopft. Da sind sie! Was wollen sie den heute schon wieder holen? Gestern waren es die Kühe. Vorgestern war's das Bettzeug. Vorvorgestern Pflug, Egge und Sämaschine. Pferde, Geschirr und Wagen holten sie am ersten Tag. Was holen sie sich heute? Es kann doch nicht wahr sein, daß nun die Mastschweine dran sind, wovon werden wir leben, wenn sie auch die Mastschweine wegnehmen! Doch es war die Wahrheit, sie holten sich auch die gemästeten Schweine. (Birkner: 161-162) 


\section{Das Bild des Zigeuners : das Gegenüber}

Birkners Die Tatarenpredigt stellt das multiethnische und -konfessionelle Bild Siebenbürgens dar. Trotzdem tritt im Roman das Bild des Zigeuner besonders hervor, eine Tatsache, die mit dem konkreten sozial-historischen Hintergrund in Beziehung steht: es ist die kleine Zeitspanne, als den Zigeunern zum ersten Mal in ihrer Geschichte von den Behörden großeAufmeksamkeit geschenkt worden ist.

Wer sind die Zigeuner Rumäniens? Der rumäniendeutsche Journalist und Zigeunerforscher Franz Remmel definiert die Zigeuner als Volk, mit dem wir seit Jahrhunderten in Nachbarschaft zusammenleben:

Überleitend sei festgestellt, dass wir mit den 'Zigeunern' hierzulande ein Volk verstehen, das auf rumänischer Heimstätte erstmals 1385 Erwähnung findet - die Roma also zu den historisch gewachsenen Minderheiten des Landes zählen. ${ }^{20}$ In Siebenbürgen erstmals 1416 erwähnt, das Gebiet gehörte zu Ungarn. ${ }^{21}$

Das Dokument, in dem die Zigeuner als "ethnische Minderheit" erwähnt werden, spezifiziert, dass diese bis 1856 in der Moldau und Walachei den sozialen Status als Robi, als "einen sklavenartigen sozialen Zustand"22 hatten.

$\mathrm{Zu}$ ihrer ihre Bezeichnung sei gesagt, dass es im deutschsprachigen Raum mehrere Begriffe parallel verwendet werden bzw. Sinti, Roma und Zigeuner, das letzte wird allerdings als Stigma eingeschätzt. Beziehen wir uns aufdie Roma Rumäniens, so stehen die Sachen ganz anders; laut Remmel möchte ein

${ }^{20}$ Remmel, Franz: Eigendarstellung und Gegenüber. Der Weg zur EU mit den Roma im Reisegepäck. In: M.Sass/H. Uerlings/I.K. Patrut: Europa und seine Zigeuner. Universitätsverlag Sibiu/Hermannstadt 2007, S. 119.

${ }^{21}$ Remmel, Franz: Der Turm zu Babel. Ein Mosaik zur rumänischen Romagesellschaft. InterGraf Verlag Resita, 2004, S. 29.

${ }^{22}$ Remmel, Franz: Vgl. Fußnote 21, S. 119. 
großer Prozentsatz der Zigeuner nicht als Roma bezeichnet werden:

Der Bulibasse von Crăciuneşti erklärt öffentlich: Sagst du mir Rom, dann beleidigst du mich. Nennst du mich 'Zigeuner', dann sprichst du mir zu Herzen. (...) Sinti und Roma sind mehr oder weniger politisch ausgehandelte Kunstbegriffe political correctness, welche die Bürgerrechtbewegung deutscher Sinti und Roman durchgesetzt hat. ${ }^{23}$

In der vorliegenden Arbeit werden bewusst beide Bezeichnungen, Roma und Zigeuner, parallel verwendet, einerseits der offizielle Begriff "Roma", andererseits die von Birkner angeführte Bezeichnung "Zigeuner".

Derselbe Franz Remmel hebt hervor, dass es über die Herkunft der Roma mehrere Theorien gibt und dass die Zigeuner Rumäniens aus Indien stammen würden. Seine Behauptung fußt auf sprachliche Gemeinsamkeiten zwischen der RomaniSprache und dem Sanskrit.

Im Mittelpunkt der vorliegenden Arbeit steht das Bild des Zigeuners als ethnische Minderheit, wie es in dem Roman Die Tatarenpredigt von Birkner vorhanden ist. Diese ethnische Gruppe erlebte vor dem zweiten Weltkrieg keine günstige Zeiten, ihr "sklavenartiger" Status wird auch im 20. Jh. beibehalten, man sprach sogar von einer "Robie des 20.Jhs." ${ }^{24} \mathrm{Als} 1940$ Mihai Antonescu in Rumänien an die Macht kam, wurden die Roma nach Transnistrien deportiert. Doch nach der AntonescuDiktatur, ab dem 23. August 1944, folgte die sogenannte "Diktatur des Proletariats" 25 bzw. die erste Entwicklungsphase der Gründung einer neuen Gesellschaftsordnung auf "sozialistischer" Basis, in der die Roma - "ein Segment des Proletariats, zugleich die 'gesündesten Elemente' im Klassenkampf-von den neuen Machthabern geschätzt"26 - wurden. Bis zu dem Zeitpunkt

${ }^{23}$ Ebenda, S. 131.

${ }^{24}$ Remmel, Franz: Vgl. Fußnote 22, S. 39.

${ }^{25}$ Ebenda, S. 40.

${ }^{26}$ Ebenda, S. 40). 
die unterste Schicht der Gesellschaft, waren die Zigeuner diejenigen "die von der damaligen PMR (Rumänische Arbeiterpartei), Vorläufer der PCR (Rumänische Kommunistische Partei) hochgespielt, propagandistisch ausgeschlachtet, politisch manipuliert und dann abgenabelt..." ${ }^{27}$ Die RKP verwendete die Roma "bis die Säcke der Propagandisten im Trockenen waren." 28 In dieser Zeitspanne vollzog sich eine "Umkehrung der Werte." ${ }^{29}$ Wie schon oben angeführt, verloren die Siebenbürgen Sachsen durch die Enteignung ihr Hab und Gut zugunsten der Zigeuner. Hatten die Roma in der Vergangenheit eine gute Beziehung zu den Sachsen, die ihre Arbeitsgeber gewesen waren, gehabt, die Enteignung brachte nicht nur eine materielle, sondern auch eine "emotionale Scheidung von den Roma." 30 Das Proletariat hatte das Sagen, die Sachsen wurden aus ihren eigenen Häusern verdrängt. Die Roma, die über Nacht hochgekommen waren, besetzten die sächsischen Häuser, eine Tatsache, die "von den Geschädigten als tiefes Unrecht empfunden" 31 wurde und nachhaltige Spannungen zwischen den beiden Ethnien mit sich brachte.

Diese Zeitspanne schildert Andreas Birkner in der Tatarenpredigt. Episoden aus dem Zusammenleben jener Jahre, zwischen Rumänen, Ungarn, Deutsche und Roma stehen "für einen Abschnitt aus der Geschichte der RKP. Denn die Roma agierten auch dazumal nicht eigenmächtig. Sie agierten als manipulierte der RKP." ${ }^{32}$ Es ist eine neue "Welle", die bis zu dem Zeitpunkt unvorstellbare gesellschaftliche Veränderungen mit sich gebracht hatte, eine "sozialistische Diktatur der Roma"33 war ausgebrochen; Ein ungarischer Zigeuner wurde zum

${ }^{27}$ Ebenda, S. 40).

${ }^{28}$ Remmel, Franz: Vgl. Fußnote 21, S. 120.

${ }^{29}$ Remmel, Franz: S. 41.

${ }^{30}$ Ebenda, S. 41.

${ }^{31}$ Ebenda, S. 41.

${ }^{32}$ Ebenda, S. 43.

${ }^{33}$ Remmel, Franz: Vgl. Fußnote 22, S. 41 
Bürgermeister von Pretai ernannt. Während die Sachsen und die Rumänen noch eine gemeinsame Sprache fanden, entwickelte sich zwischen Sachsen und Roma eine starke Abneigung; Angst herrschte in der ganzen dörflichen Gemeinschaft: "Man fürchtete sich, bei Tag, erst recht bei Nacht, das Haus zu verlassen." 34

Aufgrund des dargestellten sozial-historischen Kontextes konstruiert Birkner, wie auch andere siebenbürgische Autoren (z.B. Heinrich Zillich, Erwin Wittstock u.a.), das Bild des Zigeuners, das er aus näherer Umgebung, aus Siebenbürgen, als "das Fremde" in seiner "bekannten" "eigenen" Umgebung erlebt hat, wobei das aufgestellte Bild ambivalent ausfällt. Obwohl der Autor in dem Darstellungsbild differenziert vorgeht, nicht nur Negatives zu gestalten, wird nicht deutlich klar, dass er als Anliegen betrachtet, das Romanbild von Ideologisierungen und Stereotypisierungen zu befreien. Ein Feindbild ${ }^{35}$ so wie es in der Fachliteratur definiert wird, konturiert sich nicht gänzlich, denn in einigen Aspekten wird auch das Gemeinsame und Verbindende, das Allgemein-Menschliche dargestellt. Dazu kann beispielsweise die Begegnung zwischen Malmkroger und dem Bulibascha, die Charakterisierung des Bulibascha oder der Frauen im Roman erwähnt werden, aber auch das gemeinsame Schicksal der Deportation, wie es sowohl die Roma während des Krieges und die Siebenbürger Sachsen ab Januar 1945 erlebt haben.

${ }^{34}$ Ebenda, S. 42

${ }^{35}$ Mit Feindbild wird im Allgemeinen ein soziales Deutungsmuster gegenüber anderen Menschen, Menschengruppen (insbesondere Minderheiten), Völkern, Staaten oder Ideologien bezeichnet, das auf einer Schwarz-Weiß-Sicht der Welt (Dichotomie, Dualismus) beruht und mit negativen Vorstellungen, Einstellungen und Gefühlen verbunden ist. Typisch für ein Feindbild ist, dass im Anderen bzw. Fremden das Böse gesehen wird und diesem negativen Bild kontrastierend ein positives Selbstbild bzw. Freundbild gegenübergestellt wird. (http://de.wikipedia. org/wiki/Feindbild: 8.11.2014) 
Die Romagestalt, die uns schon im ersten Kapitel des Romans begegnet ist der Bullibascha, dem ein fast ausschliesslich positives Bild gewidmet ist:

Der Bullibascha - der jüngste der Zeltzigeuner war er nicht mehr, Bart und Kopfhaar, das ihm beide Schultern bedeckte, ergraut, der Ledergürtel hing ihm unterm Bauch - der Pferdehandel war schon immer sein Geschäft gewesen. (...) Der Bullibascha war kein Kesselflicker, der von Hof zu Hof zog und seine Künste anbot. Keiner also von den Zegunen mit dem geschulterten Sack, in dem sie auflasen, was sie fanden, dem man nachsah, bis er das Gassentor hinter sich zugezogen hatte. (...) Den Bullibascha trieb es vielmehr von Jahrmarkt zu Jahrmarkt, landau und landab, er handelte Pferde, stolze Rosse und dürre Klepper, Rappen und Schimmel, eitle Lipizzaner und schwere Nonius und alle Kreuzungen hin und her, eselsköpfige Heimtücker und kleinhirnige Allerweltsrösser oder, selten genug, glutäugige Araber, er kaufte und verkaufte, verstand sich auf den Gang der Pferde, auf die wippenden Gelenke, zog an den Ohren und las ihren Geburtsschein aus dem aufgerissenen Maul, und wie aus einer Mähre ein feuriges Roß wurde, auch davon hätte er manches zu berichten gewußt, selbst die Kunst, wie aus einem Fuchs über Nacht ein Rappe zu machen ist, war ihm sicherlich nicht unbekannt, wenn er auch bei Eid und Fluch geleugnet hätte, sie jemals praktiziert zu haben. (Birkner: 8)

Im oben angeführten Abschnitt wird nicht explizt gesagt, sondern nebenbei suggeriert, dass der Bulibascha auch ein Pferdedieb sein könnte; dazu verwendete Birkner eine stereotypenhafte Charakterisierung über die Diebstählereien der Zigeuner.

Nach der äußerlichen Beschreibung des Bulibascha, der in den Bauernhöfen nie anzutreffen war, wird auf eine veränderte Beziehung zwischen den Sachsen, vertreten durch Malmkroger, und den Roma, vertreten durch den Bullibascha, hingewiesen: "Es war das erste Mal in seinem Leben, daß er einem Zeltzigeuner einen Krug Wein auf den Tisch stellte.” (Birkner: 12) Bullibascha will dem Sachsen Gold geben, um es zu verstecken, damit der Zigeuner es zurückbekommt, wenn er aus der 
Deportation zurückkommt. Jeder von den beiden hat sich ein mentales Bild vom Anderen konturiert: Malmkroger denkt über sein Gegenüber, es sei klug: "Ein solcher Bullibascha ist klüger als zehn Feldherren." (Birkner: 13) Demgegenüber sieht der Zigeuner in Malmkroger einen Menschen, dem man vertrauen kann, mit dem man keine Papiere unterschreiben muss, um das Versprochene einzuhalten. Positiv dargestellt ist auch Paraskiva, die Mutter des Zigeuner Rudi, sie war "eine wegen ihrer Flinkheit sehr gesuchte Tagelöhnerin" (Birkner: 20). Wegen ihrer Schönheit und Reinheit wurde sie auch von Sachsen begehrt; ein sächsischer Lehrer, Namens Salmen Rudi, war der Vater ihres Sohnes, doch war dieser noch vor der Geburt des Kindes aus dem Dorf verschwunden und hatte in einer evangelischen Schule aus Galatz an der Donau Arbeit chlägerei von der Hochzeit hatte, die zum Scheitern der dritten Vermählung der Bulibaschaenkelin, Argentina, geführt hatte. Im Romamilieu hieß es nämlich, dass die angebotenen Silberbecher nicht genügend gewesen seien, was eigentlich stimmen könnte, denn Edelmetalle stellen für die Zigeuner "das einzig erstrebenswerte Eigentum", alles was sie erarbeitenten oder erbettelten, investierten sie in Goldmünzen oder Pokale.

Drei alte Zigeunerinnen, jede mit ihrem Sack über der Schulter, tauchten oben beim Ausschank auf. Sie kamen wohl von den Zelten oben im Langen grund. Alt alle drei, gebückt mehr unter der Last der Jahre alt unter dem Sack, schritten sie zielsicher voran, gefolgt vom Bullibascha. Das war allerdings sonderbar, denn sonst war's nämlich umgekehrt: voran schritt der Mann, der Zigeuner, gar der Bullibascha, aufrecht und fast ein wenig gebläht in seinem Herrenstolz, der sich weigerte, eine Last zu tragen - die luden sich die Weiber auf, einen Sack oder ein Kind, oder beides -, voran also schritt der Mann, und nur in seinem gebührlichen Abstand folgten ihm die Seinen, Frau und Kinder. (Birkner: 343) 


\section{Fazit}

Der durch eine realistische Schreibweise charakterisierte Roman Birkners Die Tatarenpredigt entstand in Retrospektive in Deutschland und gibt als Reflektor der Innenseite der Geschichte aussagekräftige Aufschlüsse über Begebenheiten des Beginns der kommunistischen Ära sowie über deren Wahrnehmung durch Betroffene. Beide untersuchten Bilder, sowohl das Eigenbild als auch das Bild des Gegenübers, sind mit vielen Vorurteilen behaftet. Wie Wolfgang Höpken ${ }^{36}$ hervorhebt, sind sowohl Heterotype (Fremdwahrnehmung), als auch Autostereotype (Selbstwahrnehmung) Instrumente der nationalen Identitätsschöpfung, in Birkners Roman bauen sich beide Bildarten auf Bipolarität in der Bewertung des Eigenen und des Gegenübers auf. Während das Bild des Sachsen hauptsächlich positiv, durch Überlegenheit versus seinem Gegenüber wahrgenommen wird, fällt das Romabild - wenn auch einigermaßen differenziert dargestellt - hauptsächlich negativ aus. Sachsen und 'Zigeuner' lebten in Siebenbürgen lange Zeit zusammen, bei jeder ethnischen Gemeinschaft hatte sich ein Eigen- bzw. Fremdbild herauskristallisiert, Bilder, die sich gegenseitig bedingen. Die Zigeuner leben räumlich wie sozial am Rande des Dorfes: "Sie gehörten nicht dazu, waren auch nicht grundsätzlich ausgeschlossen." (S.331, Birkner).

Birkner versucht das verbitterte gesellschaftliche siebenbürgische Bild durch treffende stilistische Mittel erträglicher zu machen, von denen wir die Ironie, stellenweise einen anekdotischen Reiz und gutmütigen Humor hervorheben.

${ }^{36}$ Wolfgang Höpken: Ethnische Stereotype in Sydosteuropa. Anmerkungen zu Charakter, Funktion und Entstehungsbedingungen. In: Das Bild des Anderen in Siebenbürgen. Stereotype in einer multiethnischen Region. Hrsg. von Konrad Gündisch/ Wolfgang Höpken /Michael Markel. Siebenbürgisches Archiv. Band 33, Böhlau Verlag: Köln/Weimar/Wien, 1998, S. 33 
Noch eine Bemerkung darf abschließend nicht unerwähnt bleiben: in Birkners Roman werden literarische Bilder dargeboten, das Ästhetische steht im Vordergrund, wobei die Persönlichkeit des Autors als Gestaltungsfaktor zu berücksichtigen ist. Daraus resultiert der persönlich-subjektive und fiktionale Charakter der literarischen Bilder, denn bei der Erforschung des außerliterarischen Kontextes, nämlich bei der Erhellung politisch-historischer Hintergründe sprachlich fixierter Fremdenbilder, handelt es sich weniger um die Rekonstruktion der historischen Gegebenheiten schlechthin - genauso wie es weniger um die Untersuchung um ihrer selbst willen geht -, sondern um die literarische Darstellung der "fremden Wirklichkeit" und der Vorstellungen von ihr. Doch ist es zu ergänzen, dass man mit Vorurteilen niemals dem Gegenüber näher kommen wird, sondern durch Toleranz, die eigentlich Birkner utopisch am Ende des Romans, bei dem Begräbnis des Tatters, durch die Anwesendheit der Pfarrer aller Konfessionen, als Möglichkeit wiedergibt.

\section{Bibliographie:}

\section{Primärliteratur}

Birkner, Andreas: Die Tatarenpredigt. Roman. Europaverlag. Wien 1973.

Birkner, Andreas: Der Brautschmuck des Sebastian Hann. Erzählungen. Herausgegeben und mit einem Nachwort von Hans Bergel. Mit einem editorischen Bericht von Stefan Sienerth. München 2002.

\section{Sekundärliteratur}

Duden: Deutsches Universalwörterbuch, A-Z. Dudenverlag, Mannheim/Wien/Zürich 1989. 
Erzse-Boitor, Kinga: Das Bild des Anderen in der rumäniendeutschen Kinder- und Jugendliteratur. Peter Lang, Frankfurt am Main, 2009.

Funda, Kiziler Emer: Die Imagologie als Arbeitsbereıch der Komparatıstık. 2012. http://www.ijoess.com/Makaleler/ 1275068110_Die\%20Imagologie\%20als.pdf: 15.10.2019 Gündisch, Konrad / Höpken, Wolfgang / Markel, Michael (Hrsg.): Das Bild des Anderen in Siebenbürgen. Stereotype in einer multiethnischen Region. Siebenbürgisches Archiv. Band 33, Böhlau Verlag: Köln/Weimar/Wien, 1998.

Hoffmann, Michael: Interkulturelle Literaturwissenschaft, Wilhelm Fink Verlag. Stuttgart 2006.

Honnef-Becker, Irmgard (Hrsg.): Dialoge zwischen Kulturen, interkulturelle Literatur und ihre Didaktik. (Diskussionsforum Deutsch. Bd. 24). Baltmannsweiler-Schneider Verlag Hohengehren 2007.

Logvinov, Michail, I.: Studia Imagologica: zwei metodologische Ansätze zur komparatistischen Imagologie 1. In: Germanistisches Jahrbuch GUS: Das Wort, 2003.

Nowotnik, Michaela: „95 Jahre Haft”. Kronstädter Schriftstellerprozess 1959: Darstellungsformen und Deutungsmuster der Aufarbeitung. In: Halbjahresschrift für südosteuropäische Geschichte, Literatur und Politik. 24. Jg., Heft 1-2/2012.

Nünning, Ansgar: Grundbegriffe der Literaturtheorie. J.B. Metzler Verlag, Stuttgart 2004.

Nünning, Ansgar (Hrsg.): Metzler Lexikon. Literatur- und Kulturtheorie. 4. Auflage. J.B. Metzler, Stuttgart-Weimar 2008.

Remmel, Franz: Eigendarstellung und Gegenüber. Der Weg zur EU mit den Roma im Reisegepäck. In: M. Sass/ H. Uerlings/ I.K. Patrut: Europa und seine Zigeuner. Universitätsverlag Sibiu/Hermannstadt 2007.

Remmel, Franz: Der Turm zu Babel. Ein Mosaik zur rumänischen Romagesellschaft. InterGraf Verlag Resita, 2004. 
Remmel, Franz: Nackte Füsse auf steinigen Straßen. Aldus, Brasov/Kronstadt, 2003.

Wahrig: Deutsches Wörterbuch. Bertelsmann Lexikon Verlag, Güterloh und München, 1986-1991.

Wilpert, Gero von: Sachwörterbuch der Literatur. Kröner Verlag, Stuttgart 1989. 